\title{
Near-Infrared Brain Volumetric Imaging Method: A Monte Carlo Study
}

\author{
Ching-Cheng Chuang, Pei-Ning Wang, Wei-Ta Chen, Tsuo-Hung Lan, Chung-Ming Chen, \\ Yao-Sheng Hsieh, Chun-Yang Wang, and Chia-Wei Sun
}

\begin{abstract}
The symptom of brain volumetric changes may provide significant biomarker to predict progressive dementia. The brain volumetric changes of prefrontal cortex are highly associated with many neurodegenerative diseases. Besides, brain atrophy reveals the expanded interhemispheric fissure and the concomitant increasing cerebrospinal fluid volume. Thus, the quantitative assessment of brain volumetric changes is an important consideration for clinical studies of neurodegenerative diseases. In this study, we first proposed an approach that uses near-infrared brain volumetric imaging to detect brain volumetric changes. The healthy, aged, and typical Alzheimer's disease (AD) brains were modeled with different characterization of brain volumetric changes from in vivo MRI data based on time-resolved 3-D Monte Carlo simulation. In the results, the significant difference of prefrontal cortex structure can be observed among healthy, aged, and $\mathrm{AD}$ brain with various source-detector separations in sagittal view. Our study shows that the near-infrared brain volumetric imaging can be an indicator of brain atrophy for clinical application of neurodegenerative diseases with patient-oriented measurement.
\end{abstract}

Index Terms-Brain atrophy, brain volumetric imaging (BVI), diffuse optical imaging (DOI), Monte Carlo simulation, nearinfrared spectroscopy (NIRS), prefrontal cortex.

\section{INTRODUCTION}

B RAIN atrophy is an irreversible brain disease that causes problems with cognitive and memory functions in many

Manuscript received March 16, 2011; revised May 10, 2011 and June 20, 2011; accepted June 22, 2011. Date of publication August 8, 2011; date of current version June 1, 2012. This work was supported in part by the Taiwan National Science Council under Grant 100-2221-E-010-004, Grant NSC 99-2221-E-010-011, a grant from Ministry of Education, Aim for the Top University Plan in National Yang-Ming University and Yen Tjing Ling Medical Foundation.

This paper has supplementary downloadable material available at http://ieeexplore.ieee.org, provided by the authors. This includes an MOV video showing dynamic photon migration witn $800 \mathrm{~nm}$ light pulse illumination. This material is $4.26 \mathrm{MB}$ in size.

C.-C. Chuang is with the Institute of Biomedical Engineering, National Taiwan University, Taipei 10617, Taiwan.

P.-N. Wang and W.-T. Chen are with the Department of Neurology, Neurological Institute, Taipei Veterans General Hospital, Taipei 11221, Taiwan, and also with the School of Medicine, National Yang-Ming University, Taipei 11221, Taiwan.

T.-H. Lan is with the Department of Medicine, School of Medicine, National Yang-Ming University, Taipei 11221, Taiwan.

C.-M. Chen is with the Institute of Biomedical Engineering, National Taiwan University, Taipei 10617, Taiwan.

Y.-S. Hsieh and C.-Y. Wang are with the Department of Photonics, National Chiao Tung University, Hsinchu 30010, Taiwan.

C.-W. Sun is with the Biophotonics Interdisciplinary Research Center and Institute of Biophotonics, National Yang-Ming University, Taipei 11221, Taiwan, and also with the Department of Photonics, National Chiao Tung University, Hsinchu 30010, Taiwan (e-mail: chiaweisun@ym.edu.tw).

Color versions of one or more of the figures in this paper are available online at http://ieeexplore.ieee.org.

Digital Object Identifier 10.1109/JSTQE.2011.2163927 diseases, such as mild cognitive impairment (MCI), Alzheimer disease (AD), multiple sclerosis, schizophrenia, alcoholism, dementia, etc. [1]-[6]. The neuropathological process of brain atrophy involves progressive biochemical and structural changes that begin at the cellular and synaptic level, and ultimately culminate in neuronal death, white matter (WM) and gray matter (GM) degeneration [2]. The loss of neurons and synapses in the cerebral cortex and subcortical regions results in gross atrophy of the affected regions, including degeneration in the hippocampus, temporal lobe, parietal lobe, and frontal cortex [7]-[12].

The quantitative assessment of brain volumetric changes is becoming an important consideration in monitoring the clinical outcome and treatment effects. The reason is that recent considerable advances in neuroimaging and computer technology have allowed the study of brain volumetric changes in vivo, which could provide an accurate, reproducible, and quantitative measure for assessing brain volumetric changes. Several neuroimaging modalities, such as MRI, computed tomography (CT), and positron emission tomography (PET), could be used to provide the noninvasive investigation for human brain imaging and then to identify regional atrophy characteristics with their particular advantages [2], [3], [6], [13], [14]. However, the limitation of a large size of instruments cannot provide diagnosis with patient-oriented measurement. Contrarily, near-infrared spectroscopy (NIRS) or diffuse optical imaging (DOI) has several benefits such as noninvasive, less expensive, nonionizing radiation imaging, real-time measurement, compact implementation, long-time monitoring, and easy operation with high temporal resolution $(\sim \mathrm{ms})$ and adequate spatial resolution $(\sim \mathrm{cm})$ for continuously recording oxy- and deoxyhemoglobin changes of brain. Furthermore, the optical method offers completely patient-oriented measurement. Recently, the NIRS technique has been shown to be a noninvasive functional neuroimaging technique for measuring local changes in concentrations of oxyhemoglobin and deoxyhemoglobin with dual-wavelength nearinfrared illumination [15]-[19].

The most significant symptom of neurodegenerative diseases that can be imaged by MRI is the volumetric changes with brain atrophy, especially for early diagnosis of brain atrophy [20], [21]. Brain atrophy causes the volume of cerebrospinal fluid (CSF) layer to expand. The previous studies show that the light-guiding effect occurs in the CSF layer of human brain with near-infrared light illumination [22]-[24]. For investigation of individual difference in brain volumetric changes with NIRS, three brain models from different stages of brain atrophy were modeled to implement Monte Carlo simulated with various source-detector separations. As mentioned earlier, the light-guiding effect occurred in the CSF layer of human brain. The presence of a relatively clear layer such as CSF that has both 
low scattering and absorption coefficients has been shown especially to alter the light propagation in the head. This phenomenon cannot be portrayed by diffusion approximation method because the CSF reveals low scattering property but it can be observed in the Monte Carlo simulation [24], [25].

In this paper, we offer an approach of 3-D brain modeling with image segmentation processes from in vivo MRI T1 data, in order for a comparison of diffuse optical signal among normal, aged, and AD subjects with different characterization of brain volumetric changes. To our knowledge, this is the first study to show the structural monitoring for brain volumetric change detection by using near-infrared DOI via various source-detector separations. Although the penetration depth of near-infrared light is limited by strong scattering in human brain, our simulation result indicates that the optical method still allows detecting the volumetric changes of cerebral cortex with brain atrophy that caused the concomitant expanded CSF volume with the WM and GM degeneration, especially in interhemispheric fissure of prefrontal cortex. Because the expanded CSF volume offers light-guiding channels, our optical measurement could be a great tool to detect brain volumetric changes for patientoriented neurodegenerative diseases diagnosis.

\section{DOI FOR STRUCTURAL CHARACTERIZATION OF BRAIN}

In our study, three brain models with different atrophy statuses are used for Monte Carlo simulation. The subjects include a healthy control (male, age: 40), an elder volunteer (female, age: 62), and a typical AD patient (female, age: 80 ). All subjects were recruited and MRI imaged in Taipei Veterans General Hospital. In clinical protocol, the severity of dementia was evaluated with clinical dementia rating (CDR) scale, and the AD patient was mild AD with a CDR score of 1 . The healthy and aged controls were cognitively normal. However, the aged case was evaluated with normal age-related brain atrophy. In this paper, the simulation was achieved by four steps: 1) realistic optical modeling of human brain based on image segmentation processes by utilizing in vivo MRI T1 data; 2) the healthy, aged, and typical AD brains were modeled for Monte Carlo simulations and all the photon paths were traced for photon migration analysis; 3) 1-D images were obtained via source-detector separations with three different views for structural characterization of brain; 4) the normalization process is utilized to reduce the individual factor.

Fig. 1 shows the modeling process of 3-D human brain from in vivo MRI images. First, the clinical MRI T1 scan offers 92 axial slices as in Fig. 1(a). The images were segmented into five layers of brain tissue as scalp, skull, CSF, GM, and WM. The 3 -D brain image contains $256 \times 256 \times 92$ voxels and each voxel size is $1 \times 1 \times 1 \mathrm{~mm}^{3}$ that corresponds to the resolution of 3-D MRI image. First, the scalp and skull layers were segmented by means of edge detection and region growing as shown in Fig. 2(b) [26]-[28]. Then, the probabilistic framework was applied to classify CSF, GM, and WM layers with unified segmentation, which was performed by fitting a mixture of Gaussians (MOG) model with prior information of deformable tissue probability maps [29] (as shown in Fig. 1(c), (d), and (e), respectively). Fig. 1(f) demonstrates a 2-D five-layer brain structure after segmentation processes. Finally, Fig. 1(g) and (h) shows the 3-D MRI image and the corresponding optical

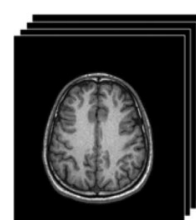

(a)

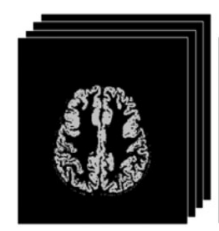

(d)

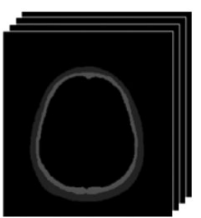

(b)

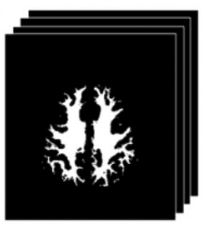

(e)

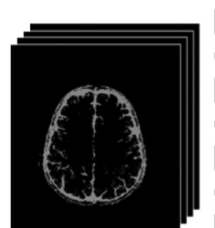

(c)

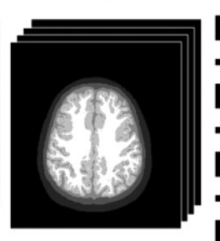

(f)

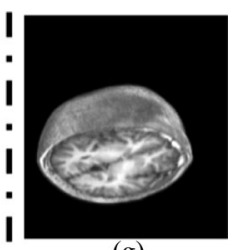

(g)

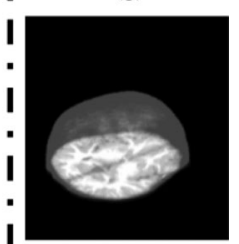

(h)
Fig. 1. Modeling process of 3-D human brain from in vivo MRI T1 images (a) 2-D anatomical MRI slice. (b) Segmentation of scalp and skull. (c) Segmentation of CSF layer. (d) Segmentation of GM. (e) Segmentation of WM (f) 2-D optical brain model in a five-layer brain structure. (g) 3-D MRI T1 image. (h) 3-D optical brain model.

brain model that was performed in Monte Carlo simulation. In the simulations, an 800-nm wavelength light source was applied for illumination of healthy, aged, and typical AD brains. The reduced scattering coefficient $\mu_{s}^{\prime}$, absorption coefficient $\mu_{a}$, scatters' radius, refractive indices of background, and scatters of brain tissues are presented in Table I [30]-[32].

The flowchart of the simulation process is shown in Fig. 2. As mentioned earlier, the volume of CSF was increased with the atrophy of GM and WM. The penetrated photons could be guided along the CSF layer because of its low scattering property. This phenomenon can be used for structural characterization of brain with NIRS/DOI measurement. Monte Carlo modeling is a method that can be used to simulate photon interaction in turbid tissue [30], [33], [34]. The Monte Carlo algorithm we used is represented as the dashed line area in Fig. 2. In this study, the point source was used, which means that all the photons start to emit at the same direction. If the photon propagates through a voxel boundary, the step size would be modified as $s=-\ln (\xi) / \mu_{t}$, where $\xi$ is a sampled value of a uniform random variable within the interval $[0,1]$ and $\mu_{t}$ is an extinction coefficient. If the refractive indices are different between two adjacent voxels, the transmission or reflection then occurs alternatively. In addition, Snell's law and Fresnel reflection formulas were applied at each boundary. A scattering event, a new step size, deflection angle, and azimuthal angle were calculated for each photon. Generally, the behavior of photon migration in brain can be decided by two steps: 1) the mean free path of a scattering/absorption event and 2) the probability density function of scattering angle. The absorption and scattering properties of a sphere can be described by the Mie theory that has been available in previous studies [35]. All of the photons are traced and recorded for photon migration analysis and imaging.

In the simulation, the light source and detectors were placed on the surface with three cross-sectional arrangements as transverse, sagittal, and coronal views (as shown in Fig. 3). The source-detector separations of the transverse and sagittal views were $1-5 \mathrm{~cm}$ with $1-\mathrm{cm}$ intervals. In the coronal view, the source was put on the top of the middle head and the detectors were located on right sides of the source. The source-detector separations were also placed from 1 to $5 \mathrm{~cm}$ with $1-\mathrm{cm}$ intervals. In 


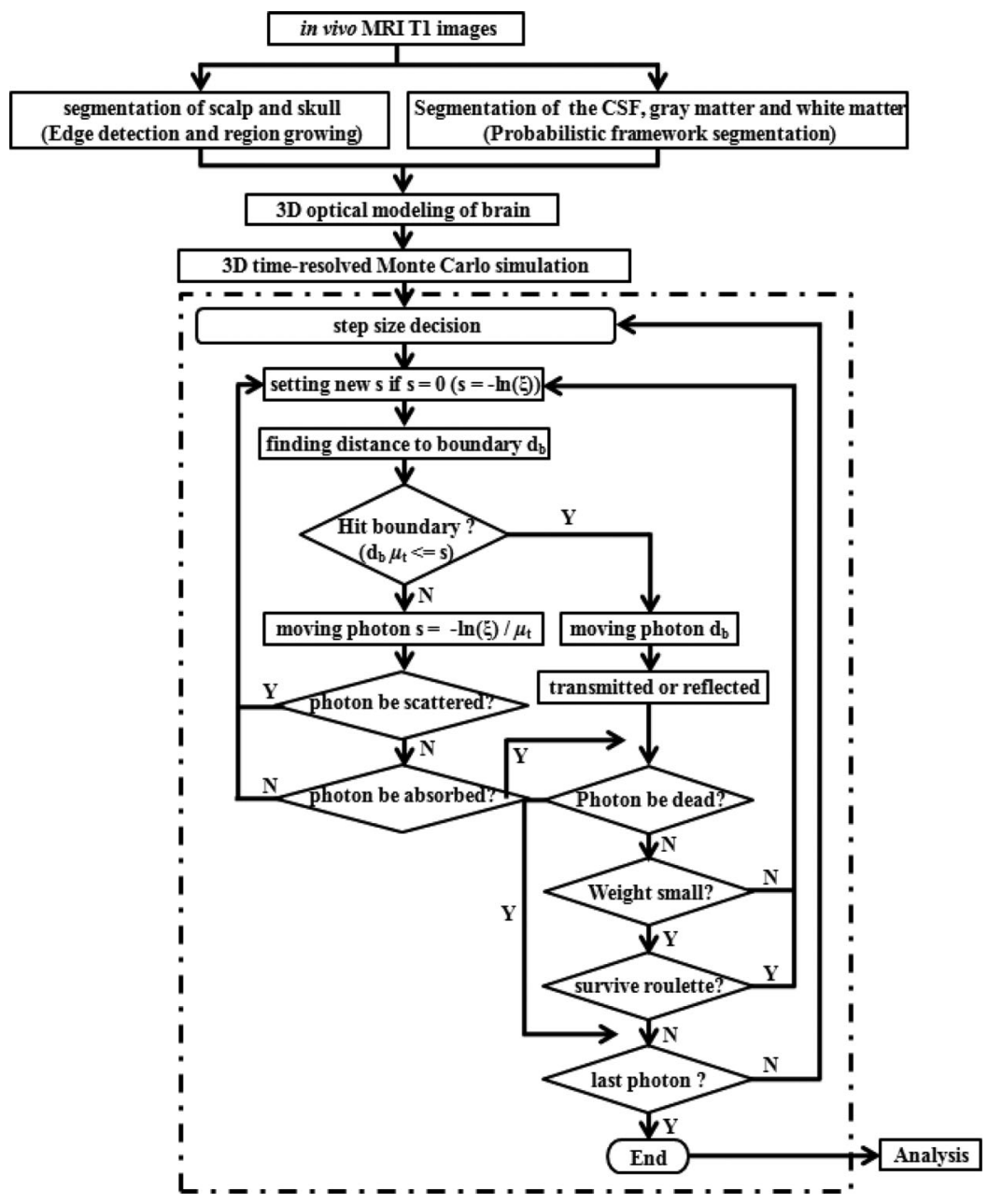

Fig. 2. Flowchart of the brain modeling process by means of the Monte Carlo algorithm with MRI data.

TABLE I

Optical Properties of Brain TISSUES IN MONTE CARLo SimUlation

\begin{tabular}{lcccccc}
\hline \hline Brain tissues & $\begin{array}{c}\mu_{\mathrm{s}}{ }^{\prime} \\
\left(\mathrm{cm}^{-1}\right)\end{array}$ & $\begin{array}{c}\mu_{\mathrm{a}} \\
\left(\mathrm{cm}^{-1}\right)\end{array}$ & $\begin{array}{c}\text { Refractive index of } \\
\text { background }\end{array}$ & $\begin{array}{c}\text { Refractive index of } \\
\text { scattering particles }\end{array}$ & $\begin{array}{c}\text { Radius of scattering } \\
\text { particles }(\mu \mathrm{m})\end{array}$ & $\begin{array}{c}\text { Anisotropy factor } \\
(\mathrm{g})\end{array}$ \\
\hline Scalp & 19 & 0.18 & 1.4 & 1.565 & 10 & 10 \\
Skull & 16 & 0.16 & 1.4 & 1.565 & 10 & 0.92 \\
CSF & 2.4 & 0.04 & 1.331 & 1.565 & 10 & 0.92 \\
Gray matter & 22 & 0.36 & 1.36 & 1.565 & 1.56 & 0.92 \\
White matter & 91 & 0.14 & 1.38 & -- & -- & 0.92 \\
\hline Air & -- & -- & 1 & & - \\
\hline \hline
\end{tabular}

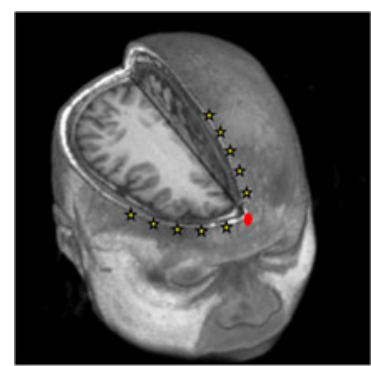

(a)

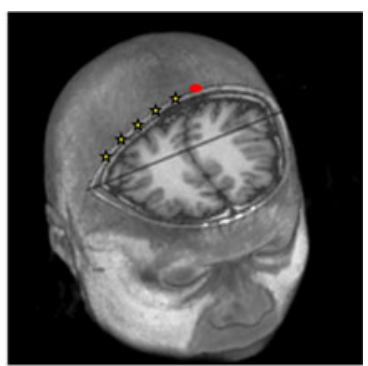

(b)

Fig. 3. Source-detector separations on human head arranged as (a) transverse and sagittal views, and (b) coronal view.
Fig. 3, the red point indicates the light source positions and the yellow stars represent the positions of detectors.

\section{RESULTS}

Fig. 4 shows the transverse, sagittal, and coronal cross sections of healthy, aged, and AD brain models.

In Fig. 4, different atrophy statuses can be seen, especially in the region of interhemispheric fissure. Besides, the atrophy of aged and AD brains and the expanded CSF volume can be obviously observed in all cross-sectional views. The characteristics are strongly correlated with the behavior of photon migration, 


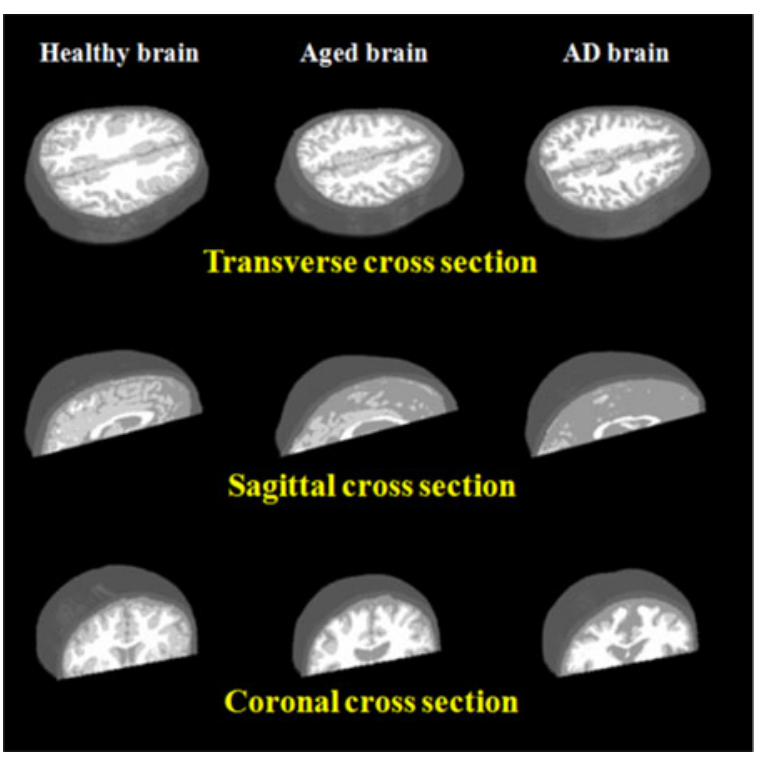

Fig. 4. Source-detector separations on human head arranged as (a) transverse and sagittal views, and (b) coronal view.

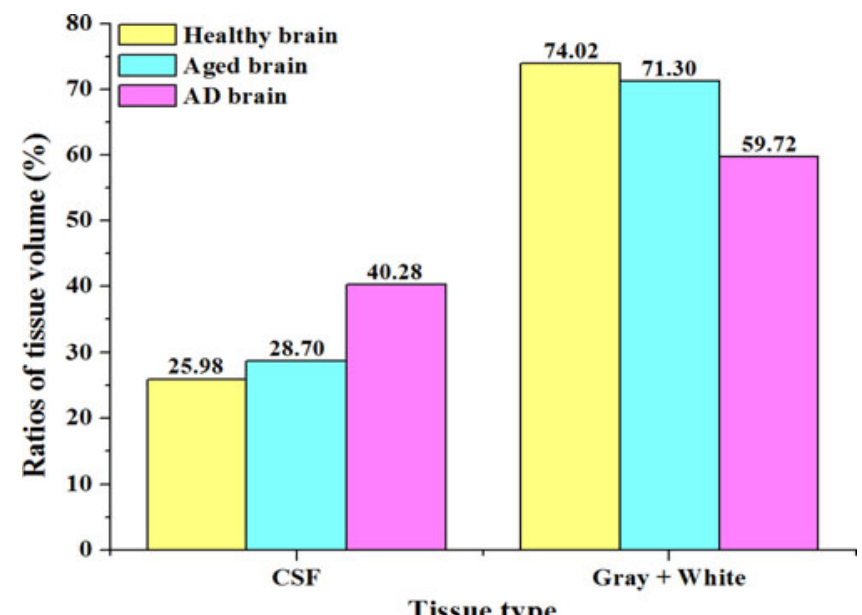

Fig. 5. Ratios of tissue volume in healthy, aged, and AD brains.

implying that the structural information related to brain atrophy could be detected by brain volumetric imaging (BVI).

For quantitative analysis, the ratios of tissue volume in three brain models are presented in Fig. 5. Apparently, the AD brain reveals the largest volume of CSF and the smallest volume of GM and WM in the group. The comparison illustrates the structural difference of atrophy status among healthy, aged, and AD brains.

Fig. 6 demonstrates the trajectories of all $2 \times 10^{7}$ penetrated diffuse photons in the transverse, sagittal, and coronal cases of both brain structures. In the healthy brain, the light can reach to WM layer ( $\sim 3 \mathrm{~cm}$ depth) after $100 \mathrm{ps}$ in transverse and coronal views. The deeper penetration can be seen in aged and AD brains because of the atrophy structure. The result implies that the traveling channel of CSF volume guides photon, and therefore, the BVI can catch the structural information of brain atrophy.

In our simulation, all photon-passed voxels were recorded for photon migration analysis. The CSF light-guiding effect can be easily observed in the supplementary movie file, which shows the dynamic photon migration with 800-nm light-pulse illumination through the healthy, aged, and AD brain models. Three cross-sectional views are demonstrated as transverse, sagittal, and coronal. The supplementary material is of $4.16 \mathrm{MB}$ and is available online at www.ieexplore.ieee.org.

Fig. 7(a)-(c) shows the intensity mapping of the detected photon with various source-detector separations in transverse, sagittal, and coronal views. Fig. 7(d)-(f) shows the intensity curves via source-detector separations. The received intensities decrease while source-detector separations increase in all cross sections. In the result, we can see that the 1-D images of sagittal views are significantly different among three brains. In Fig. 7(e), the discontinuous intensity decay results from the interhemispheric fissure in aged and AD brains. The CSF expansion of aged and AD brains causes the slow and smooth intensity to decrease, as in Fig. 7(f).

The sagittal view presents significant differences among three brains. In our previous study, the source-detector separation is usually optimally set as $\sim 3 \mathrm{~cm}$ for NIRS measurement [30]. Fig. 8 shows the normalized intensity curves via source-detector separations from 1 to $3 \mathrm{~cm}$ in sagittal view. $I_{d 1}, I_{d 2}$, and $I_{d 3}$ are the detected intensities with separations of 1,2 , and $3 \mathrm{~cm}$, respectively. Because detector 1 (with separation $1 \mathrm{~cm}$ ) receives the major backscattered intensity from scalp and skull layers, $I_{d 1}$ is treated as the baseline signal for individual variation reduction. Fig. 8 shows the normalized attenuation in all brain models. The significant difference can be seen among three brains. Furthermore, we define an index of attenuation to quantitatively classify the atrophy degree in Fig. 9. The index strongly corresponds to the volume ratio of the CSF layer in Fig. 5. In this result, the index of attenuation can be used for characterization of brain atrophy.

\section{DISCUSSION AND CONCLUSION}

Optical brain imaging continues to provide new and remarkable insights on how diseases cause impact on the human brain. Although the medical imaging systems such as MRI and PET can provide the diagnosis with structural imaging of human brain, the techniques have the main drawback of requiring large apparatus with space constraint of operation that caused difficulties for patient-oriented and bedside diagnosis and treatment. In addition, MRI chamber causes it difficult or impossible to apply to neonates, children, old persons, and claustrophobia patients. Our result indicates that the BVI technique may offer a solution for brain structure detection. In previous studies, the optical techniques were all used to measure the cortex oxygenation for functional brain imaging. Our result points out that the brain atrophy can be structurally detected by using BVI.

In Fig. 4, the 3-D geometries show the structural characteristics in all cross sections among healthy, aged, and AD brains, especially in the region of the interhemispheric fissure and the CSF volume. The result indicated that this optical measurement offers a good potential to detect the structural change of brain atrophy in clinical applications. In video 1 , we can observe that the diffuse photons are partially guided along the CSF layer, especially in interhemispheric fissure of prefrontal cortex. Thus, the mean free path of a diffuse photon in atrophy brains is deeper than in healthy brains. However, the mean free path of photon propagation in the tissue depends on source-detector separations in diffuse optics. Although the geometries of brain vary 


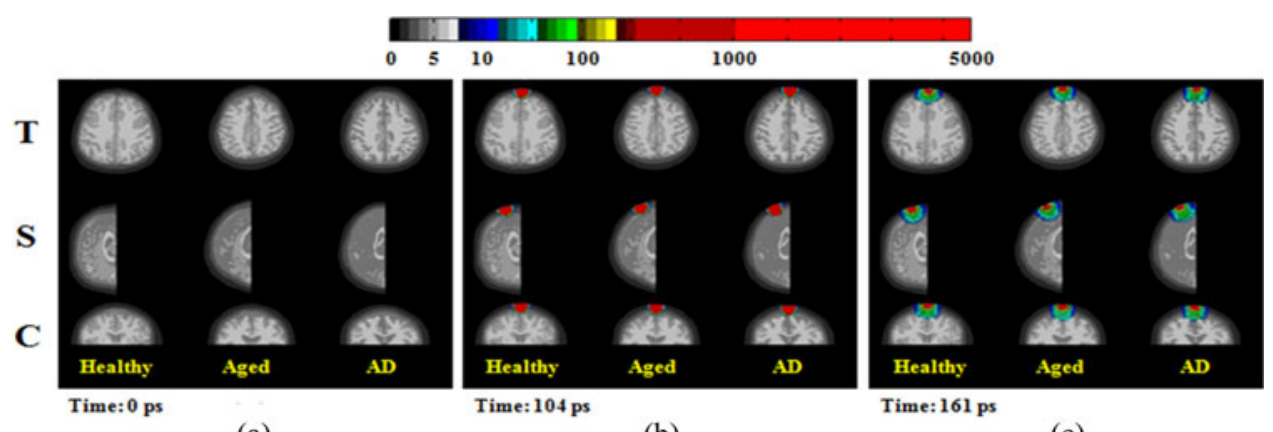

(a)

(b)

(c)

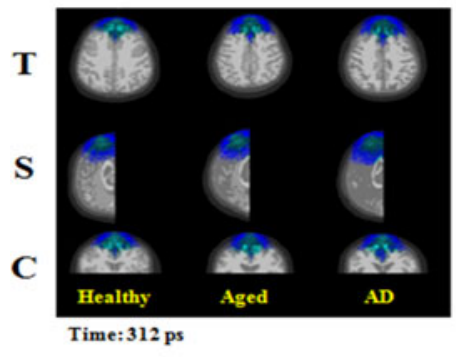

(d)

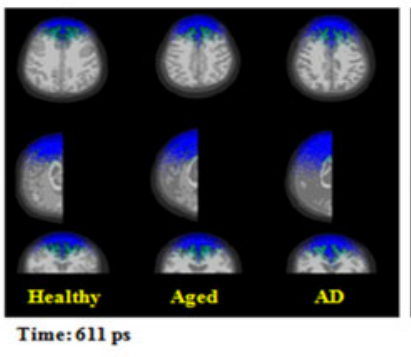

(e)

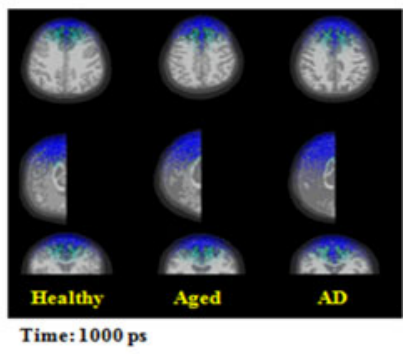

(f)

Fig. 6. Dynamics of photon migration in Transverse (T), Sagittal (S), and Coronal (C) views with 800-nm light illumination: $(a)$ at time $=0 \mathrm{ps}$, (b) at time $=$ $104 \mathrm{ps}$, (c) at time $=161 \mathrm{ps}$, (d) at time $=312 \mathrm{ps,} \mathrm{(e)} \mathrm{at} \mathrm{time}=611 \mathrm{ps}$, and (f) at time $=1000 \mathrm{ps}$.

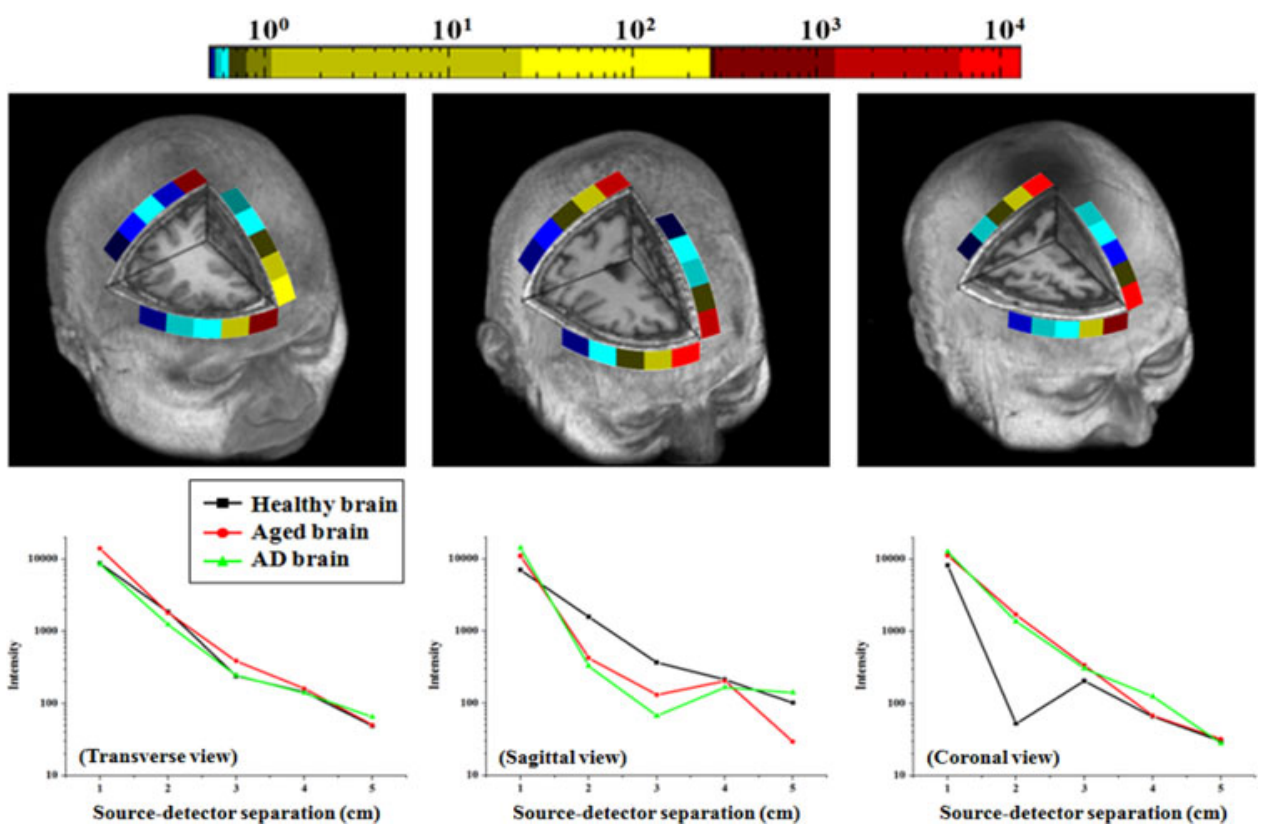

Fig. 7. Detected intensities via various source-detector separations in healthy, aged, and AD brains. (a) 1-D imaging of healthy brain in transverse, sagittal, and coronal cross sections. (b) 1-D imaging of aged brain in transverse, sagittal, and coronal cross sections; (c) 1-D imaging of AD brain in transverse, sagittal, and coronal cross sections. (d) Detected intensity via source-detector separation in transverse view. (e) Detected intensity via source-detector separation in sagittal view. (f) Detected intensity via source-detector separation in coronal view.

considerably, the source-detector separations were fixed as 1$5 \mathrm{~cm}$ with $1-\mathrm{cm}$ interval on all heads in this study. The results indicated that the geometries of brain bring little effect on the light propagation in brains. In the previous studies, the sourcedetector separation was usually optimized between 2 and $3 \mathrm{~cm}$ with optical measurement because the range of distance allows more photons to pass through the scalp and skull and then carry more information from the cortex. The individual variation of brain geometry could be ignored with the distance. To eliminate the individual difference and standardize the protocol, the intensity via source-detector separations in sagittal view was normalized for data calibration.

The brain atrophy is usually asymmetric and reveals the expanded CSF volume [36]-[38]. Fig. 5 shows the ratio of brain 


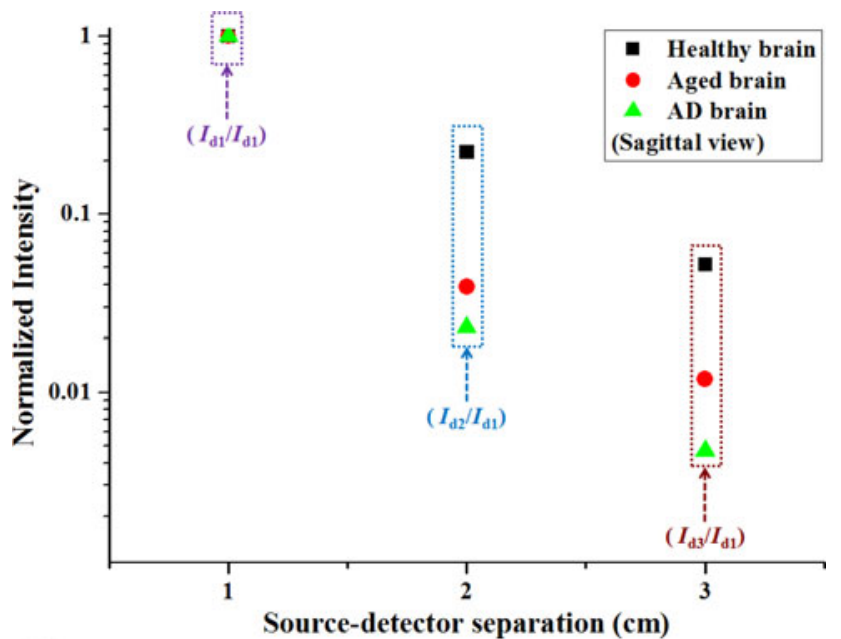

Fig. 8. Curves of normalized intensity via source-detector separations from 1 to $3 \mathrm{~cm}$ in sagittal view of healthy, aged, and AD brains.

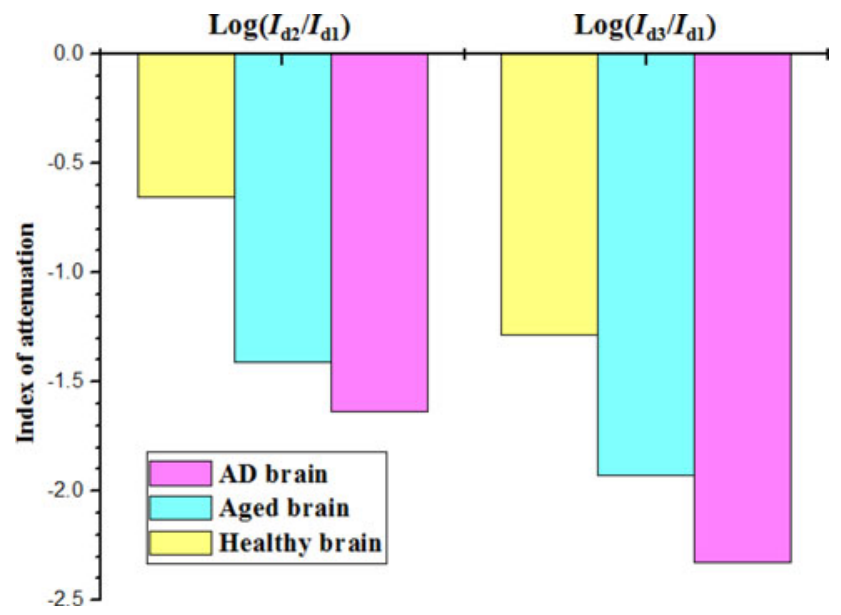

Fig. 9. Index of attenuation in healthy, aged, and AD brains' modeling. $I_{d 2} / I_{d 1}$ is the normalized intensity at $2-\mathrm{cm}$ separation and $I_{d 3} / I_{d 1}$ is the normalized intensity at 3 -cm separation.

volume of CSF, GM, and WM. In this result, the AD brain contains larger CSF and smaller GM and WM than the other cases. In Fig. 7, the brain atrophy reveals the expanded CSF volume for light guiding, which also caused the light to decrease slowly via source-detector separation in the coronal view. In other words, the larger volume of GM and WM generates stronger absorption and scattering that causes lower penetration in healthy brains. In the case of the transverse view, there is no significant difference among healthy, aged, and AD brains.

In the sagittal view, the attenuation via source-detector separation shows significant differences among healthy, aged, and AD brains. The result suggests that the expanded CSF volume permits deeper penetration of light in atrophy brains. Also, the deeper penetration of photon in the interhemispheric fissure causes the fewer backscattered photons to be received. The loss of neurons and synapses with age-related atrophy in the cerebral cortex and subcortical regions leads to degeneration in the cerebral hemispheres $(0.23 \%$ annual decline $)$, the temporal lobe $(0.28 \%$ annual decline), the frontal cortex $(0.55 \%$ annual decline), the parietal lobe ( $0.39 \%$ annual decline), and the hip- pocampus (0.3\% annual decline) [39]-[41]. The frontal cortex reveals more degeneration than the other regions. The longitudinal study of age-related brain volumetric change measurements in healthy adults has also been the subject of great interest in recent years, because the determinations of normal age-related brain volumetric changes have a role in the evaluation of both clinical-pathologic conditions and normal aging processes. Recently, some studies have indicated that the brain atrophy is identifiable in asymptomatic individuals nearly a decade before dementia. Thus, the longitudinal brain volumetric change detection may provide an indicator of early neurodegeneration for dementia diagnosis [20], [21]. Besides, some research results have shown that the prefrontal cortex atrophy is highly associated with $\mathrm{AD}$ and is even a better predictor for dementia than medial temporal lobe (hippocampus and parahippocampal gyrus) atrophy. This finding suggests that in the search of diagnostic markers for dementia, prefrontal cortex atrophy deserves more attention [42], [43]. Although the change of brain volume is small, the brain atrophy can be observed by MRI structural imaging. Hence, the optical BVI method can be a useful tool for the prefrontal cortex atrophy detection, especially for the expanded interhemispheric fissure and CSF volume estimation. The expansion of interhemispheric fissure and the concomitant expanded CSF volume cause stronger light-guiding effect with optical illumination. This implies the feasibility of brain atrophy detection based on diffuse optical measurement. The simulation results show the structural information of brain such as the expanded interhemispheric fissure of prefrontal cortex and the concomitant expanded CSF volume that can be treated as a significant factor for neurodegenerative diseases diagnosis through DOI measurement.

The brain atrophy of frontal and temporal cortices occurs in several neurodegenerative diseases. For clinical diagnosis of neurodegenerative diseases, the medical imaging systems, such as MRI, PET, and CT, even cannot provide enough specific information. But, the structure imaging still offers useful information for clinician in neurology and nerve internal medicine. In our study, neurodegenerative diseases could not be diagnosed with only brain structural monitoring by using near-infrared BVI. However, the optical method has several benefits, as mentioned earlier. Besides, the functional near-infrared optical topography offers oxygenation imaging that can be combined for clinical applications.

Although there are only three MRI brain images for healthy, aged, and AD brains' modeling, these results quite show various atrophy statuses. This study demonstrates the feasibility of structural detection by using BVI for clinical applications of neurodegenerative disease. As aforementioned, brain atrophy is identifiable in asymptomatic individuals nearly a decade before dementia. Thus, this optical method is a potiental tool for preventive medicine. In future research, we will develop a nearinfrared BVI system as our simulation scheme for the clinical study. More subjects will be recruited with different stages of brain atrophy. Also, the functional near-infrared spectroscopic imaging will be applied to both structural and functional imaging simultaneously and it is expected in application to patientoriented diagnosis. We hope that the proposed method can be applied to not only neurodegenerative diseases but also depression and schizophrenia diagnosis. 


\section{REFERENCES}

[1] D. T. Chard, C. M. Griffn, G. J. M. Parker, R. Kapoor, A. J. Thompson, and D. H. Miller, "Brain atrophy in clinically early relapsing-remitting multiple sclerosis," Brain, vol. 125, pp. 327-337, 2002.

[2] M. Pievani, F. Agosta, E. Pagani, E. Canu, S. Sala, M. Absinta, C. Geroldi, R. Ganzola, G. B. Frisoni, and M. Filippi, "Assessment of white matter tract damage in mild cognitive impairment and Alzheimer's disease," Human Brain Mapping, vol. 31, pp. 1862-1875, 2010.

[3] G. Chételat, B. Landeau, F. Eustache, F. Mézenge, F. Viader, V. de la Sayette, B. Desgranges, and J. C. Baron, "Using voxel-based morphometry to map the structural changes associated with rapid conversion in MCI: A longitudinal MRI study," NeuroImage, vol. 27, pp. 934-946, 2005.

[4] L. E. DeLisi, "The concept of progressive brain change in schizophrenia: implications for understanding schizophrenia," Schizophrenia Bull., vol. 34, pp. 312-321, 2008.

[5] J. M. Bjork, S. J. Grant, and D. W. Hommer, "Cross-sectional volumetric analysis of brain atrophy in alcohol dependence: Effects of drinking history and comorbid substance use disorder," Amer. J. Psychiatry, vol. 160, pp. 2038-2045, 2003.

[6] M. Boccardi, M. P. Laakso, L. Bresciani, S. Galluzzi, C. Geroldi, A. Beltramello, H. Soininen, and G. B. Frisoni, "The MRI pattern of frontal and temporal brain atrophy in fronto-temporal dementia," Neurobiology Aging, vol. 24, pp. 95-103, 2003.

[7] Q. Mu, J. Xie, Z. Wen, Y. Weng, and Z. Shuyun, "A quantitative MR study of the hippocampal formation, the amygdala, and the temporal horn of the lateral ventricle in healthy subjects 40 to 90 years of age," Amer. J. Neuroradiol., vol. 20, pp. 207-211, 1999.

[8] P. E. Cowell, B. I. Turetsky, R. C. Gur, R. I. Grossman, D. L. Shtasel, and R. E. Gurl, "Sex differences in aging of the human frontal and temporal lobes," J. Neurosci., vol. 14, pp. 4749-4755, 1994.

[9] C. E. Coffey, J. A. Saxton, G. Ratcliff, R. N. Bryan, and J. F. Lucke, "Relation of education to brain size in normal aging: Implications for the reserve hypothesis," Neurology, vol. 53, pp. 189-196, 1999.

[10] G. L. Wenk, "Neuropathologic changes in Alzheimer's disease," J. Clin. Psychiatry, vol. 64, pp. 7-10, 2003.

[11] X. Delbeuck, M. V. der Linden, and F. Collette, "Alzheimer's disease as a disconnection syndrome ?," Neuropsychol. Rev., vol. 13, pp. 79-92, 2003.

[12] H. J. Rosen, M. L. Gorno-Tempini, W. P. Goldman, R. J. Perry, N. Schuff, M. Weiner, R. Feiwell, J. H. Kramer, and B. L. Miller, "Patterns of brain atrophy in frontotemporal dementia and semantic dementia," Neurology, vol. 58, pp. 198-208, 2002.

[13] J. L. Whitwell, S. A. Przybelski, S. D. Weigand, D. S. Knopman, B. F. Boeve, R. C. Petersen, and C. R. Jack Jr., "3D maps from multiple MRI illustrate changing atrophy patterns as subjects progress from mild cognitive impairment to Alzheimer's disease," Brain, vol. 130, pp. 17771786, 2007.

[14] C. D. Smith, H. Chebrolu, D. R. Wekstein, F. A. Schmitt, G. A. Jicha, G. Cooper, and W. R. Markesbery, "Brain structural alterations before mild cognitive impairment," Neurology, vol. 68, pp. 1268-1273, 2007

[15] K. Sakatani, Y. Katayama, T. Yamamoto, and S. Suzuki, "Changes in cerebral blood oxygenation of the frontal lobe induced by direct electrical stimulation of thalamus and globus pallidus: A near infrared spectroscopy study," J. Neurol. Neurosurg. Psychiatry, vol. 67, pp. 769-773, 1999.

[16] T. Suto, M. Fukuda, M. Ito, T. Uehara, and M. Mikuni, "Multichannel near-infrared spectroscopy in depression and schizophrenia: Cognitive brain activation study," Biol. Psychiatry, vol. 55, pp. 501-511, 2004.

[17] M. J. Herrmann, A. C. Ehlis, A. Wagener, C. P. Jacob, and A. J. Fallgatter, "Near-infrared optical topography to assess activation of the parietal cortex during a visuo-spatial task," Neuropsychologia, vol. 43, pp. 1713-1720, 2005.

[18] H. Arai, M. Takano, K. Miyakawa, T. Ota, T. Takahashi, H. Asaka, and T. Kawaguchi, "A quantitative near-infrared spectroscopy study: A decrease in cerebral hemoglobin oxygenation in Alzheimer's disease and mild cognitive impairment," Brain Cognition, vol. 61, pp. 189-194, 2006.

[19] S. G. Diamond, T. J. Huppert, V. Kolehmainen, M. A. Franceschini, J. P. Kaipio, S. R. Arridge, and D. A. Boas, "Dynamic physiological modeling for functional diffuse optical tomography," NeuroImage, vol. 30, pp. 88-101, 2006.

[20] Y. Fan, N. Batmanghelich, C. M. Clark, and C. Davatzikosa, "Spatial patterns of brain atrophy in MCI patients, identified via high-dimensional pattern classification, predict subsequent cognitive decline," NeuroImage, vol. 39, pp. 1731-1743, 2008.

[21] B. C. Dickerson, T. R. Stoub, R. C. Shah, R. A. Sperling, R. J. Killiany, M. S. Albert, B. T. Hyman, D. Blacker, and L. de T.-Morrell, "Alzheimer- signature MRI biomarker predicts AD dementia in cognitively normal adults," Neurology, vol. 76, pp. 1395-1402, 2011.

[22] M. Firbanky, S. R Arridgez, M. Schweigery, and D. T. Delpy, "An investigation of light transport through scattering bodies with non-scattering regions," Phys. Med. Biol., vol. 41, pp. 767-783, 1996.

[23] M. Wolf, M. Keel, V. Dietz, K. von Siebenthal, H. U. Bucher, and O. Baenziger, "The influence of a clear layer on near-infrared spectrophotometry measurements using a liquid neonatal head phantom," Phys. Med. Biol., vol. 44, pp. 1743-1753, 1999.

[24] E. Okada and D. T. Delpy, "Near-infrared light propagation in an adult head model. I: Modeling of low-level scattering in the cerebrospinal fluid layer," Appl. Opt., vol. 42, no. 16, pp. 2906-2914, 2003.

[25] H. Dehghani, D. T. Delpy, and S. R. Arridge, "Photon migration in nonscattering tissue and the effects on image reconstruction," Phys. Med. Biol., vol. 44, pp. 2897-2906, 1999.

[26] P. G. van Dokkum, "Cosmic-ray rejection by Laplacian edge detection," Publication Astron. Soc. Pacific, vol. 113, pp. 1420-1429, 2001.

[27] S. A. Hojjatoleslami and J. Kittler, "Region growing: A new approach," IEEE Trans. Image Process., vol. 7, no. 7, pp. 1079-1084, Jul. 1998.

[28] A. Tremeau and N. Borel, "A region growing and merging algorithm to color segmentation," Pattern Recog., vol. 30, pp. 1191-1203, 1997.

[29] J. Ashburner and K. J. Friston, "Unified segmentation," NeuroImage, vol. 26, pp. 839-851, 2005.

[30] C. K. Lee, C. W. Sun, P. L. Lee, H. C. Lee, C. C. Yang, C. P. Jiang, Y. P. Tong, T. C. Yeh, and J. C. Hsieh, "Study of photon migration with various source-detector separations in near-infrared spectroscopic brain imaging based on three-dimensional Monte Carlo modeling," Opt. Exp. vol. 13, pp. 8339-8348, 2005.

[31] T. Li, H. Gong, and Q. Luo, "Visualization of light propagation in visible Chinese human head for functional near-infrared spectroscopy," $J$. Biomed. Opt., vol. 16, pp. 045001-1-045001-6, 2011.

[32] T. V. Dinh, Biomedical Photonics Handbook. Boca Raton, FL: CRC Press, 2003.

[33] L. H. Wang, S. L. Jacques, and L. Q. Zheng, "MCML-Monte Carlo modeling of photon transport in multilayered tissues," Comput. Methods Programs Biomed., vol. 47, pp. 131-146, 1995.

[34] M. Kirillin, I. Meglinski, V. Kuzmin, E. Sergeeva1, and R. Myllylä, "Simulation of optical coherence tomography images by Monte Carlo modeling based on polarization vector approach," Opt. Exp., vol. 18, pp. 714-724, 2010.

[35] C. F. Bohren and D. R. Huffman, Absorption and Scattering of Light by Small Particles. Hoboken, NJ: Wiley, vol. 541, 1983.

[36] S. A. Thompson, K. Patterson, and J. R. Hodges, "Left/right asymmetry of atrophy in semantic dementia: Behavioral-cognitive implications," Neurology, vol. 61, pp. 1196-1203, 2003.

[37] K. Chen, E. M. Reiman, G. E. Alexander, D. Bandy, R. Renaut, W. R. Crum, N. C. Fox, and M. N. Rossor, "An automated algorithm for the computation of brain volume change from sequential MRIs using an iterative principal component analysis and its evaluation for the assessment of whole-brain atrophy rates in patients with probable Alzheimer's disease," NeuroImage, vol. 22, pp. 134-143, 2004.

[38] J. Snowden, D. Neary, and D. Mann, "Frontotemporal lobar degeneration: Clinical and pathological relationships," Acta Neuropathol., vol. 114, pp. 31-38, 2007.

[39] C. E. Coffey, W. E. Wilkinson, L. A. Parashos, S. A. R. Soady, R. J. Sullivan, L. J. Patterson, G. S. Figiel, M. C. Webb, C. E. Spritzer, and W. T. Djang, "Quantitative cerebral anatomy of the aging human brain. A cross-sectional study using magnetic resonance imaging," Neurology, vol. 42, pp. 527-536, 1992.

[40] S. M. Resnick, A. F. Goldszal, C. Davatzikos, S. Golski, M. A. Kraut, E. J. Metter, R. N. Bryan, and A. B. Zonderman, "One-year age changes in MRI brain volumes in older adults," Cereb. Cortex., vol. 10, pp. 464-472, 2000 .

[41] R. I. Scahill, C. Frost, R. Jenkins, J. L. Whitwell, M. N. Rossor, and N. C. Fox, "A longitudinal study of brain volume changes in normal aging using serial registered magnetic resonance imaging," Arch. Neurol., vol. 60, pp. 989-994, 2003.

[42] S. Burgmansa, M. P. J. van Boxtel, F. Smeets, E. F. P. M. Vuurman, E. H. B. M. Gronenschild, F. R. J. Verhey, H. B. M. Uylings, and J. Jolles, "Prefrontal cortex atrophy predicts dementia over a six-year period," Neurobiology Aging, vol. 30, pp. 1413-1419, 2009.

[43] D. H. Salat, J. A. Kaye, and J. S. Janowsky, "Prefrontal gray and white matter volumes in healthy aging and Alzheimer disease," Arch. Neurol., vol. 56, pp. 338-344, 1999. 


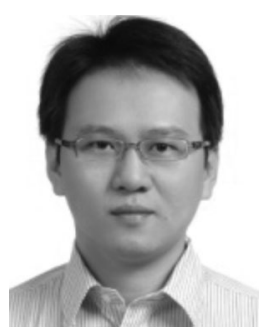

Ching-Cheng Chuang received the B.S. and M.S. degrees in biomedical engineering from Chung Yuan Christian University, Chung Li, Taiwan, in July 2003 and July 2005, respectively. He is currently working toward the Ph.D. degree in the Institute of Biomedical Engineering, National Taiwan University, Taipei, Taiwan.

He is also a Research Assistant with the Biophotonics Interdisciplinary Research Center and Institute of Biophotonics, National Yang-Ming University, Taipei, Taiwan, for neuroimaging study. His research interests include biophotonics, neuroimaging, biooptical signal/image processing, and optical device design.

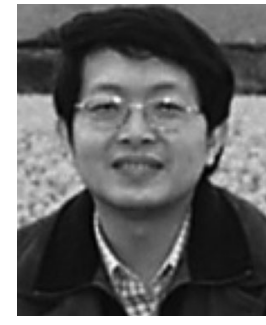

Chung-Ming Chen received the Ph.D. degree in electrical engineering in 1993 from Cornell University, Ithaca, NY.

He was involved in research on various topics such as biomedical image processing, medical imaging computer-aided detection, bioinformatics analysis, biochips analysis, and protein analysis and prediction. He is currently a Professor at the Institute of Biomedical Engineering, National Taiwan University, Taipei, Taiwan.

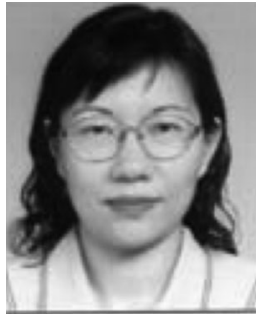

Pei-Ning Wang received the M.D. degree from the School of Medicine, National Yang-Ming University, Taipei, Taiwan, in 1990.

She was with the Department of Neurology, Taipei Veterans General Hospital. She is currently an Associated Professor in National Yang-Ming University. Her current research interests include dementia, neuroimaging, biomarkers in dementia, behavior neurology, and cognition.

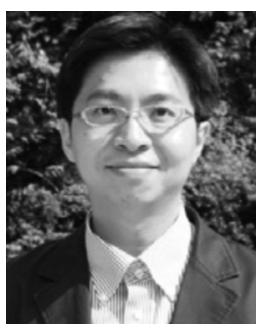

Wei-Ta Chen received the M.D. degree in 1996 from National Yang-Ming University, Taipei, Taiwan, and neurology residency training at Taipei Veterans General Hospital. He became a board neurologist in 2001 and interested in exploring the mechanism of neurological diseases by functional neuroimaging tools. In 2009, he received the Ph.D. degree from the Institute of Neuroscience, National Yang-Ming University, where he completed serial magnetoencephalographic studies in migraine disorders.

He moved to Boston in 2010 due to a two-year research appointment from Massachusetts General Hospital and Harvard Medical School for advanced studies in human functional neuroimaging. He is a Headache Specialist in Taipei Veterans General Hospital, Taipei, Taiwan, and an Assistant Professor in School of Medicine, National Yang-Ming University.

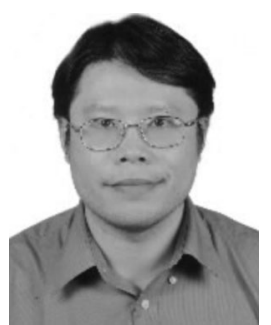

Tsuo-Hung Lan received the M.D. degree from the School of Medicine, National Yang-Ming University, Taipei, Taiwan, and the Ph.D. degree from Genetic Epidemiology Program, Department of Epidemiology, School of Hygiene and Public Health, Johns Hopkins University, Baltimore, MD.

His professional experiences are Joint-Appointed Assistant Investigator, Division of Mental Health And Substance Abuse Research, National Health Research Institutes, Taiwan (2005-present), Director of Research and Evidence-Based Medicine, Yu-Li Hospital, Taiwan (2004-present), Attending Psychiatrist, Department of Adult Psychiatry, Yu-Li Hospital, Taiwan (2004-present), Adjunct Attending Psychiatrist, Department of Psychiatry, National Taiwan University Hospital, Taiwan (2004-present), and Adjunct Assistant Professor, Department of Life Science and Institute of Genomics, National Yang-Ming University (2001-present). He has been involved in studies of genetic epidemiology in psychiatric disorders, especially in schizophrenia and bipolar affective disorder. His current research focuses on the long-term follow-up of metabolic syndrome in antipsychotics exposed schizophrenia, and the gene-environment interaction on smoking reduction among people with chronic schizophrenia. His research interests include pharmacogenomics, pharmacoepidemiology, pharmacoeconomics, and statistical model prediction on clinical outcome in people with mental illness.

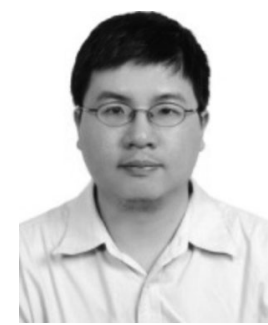

Yao-Sheng Hsieh received the B.S. and M.S. degrees from the National Taiwan University of Science and Technology, Taipei, Taiwan. He is currently working toward the Ph.D. degree at National Chiao Tung University, Hsinchu, Taiwan, where he focuses on the optical imaging methods and clinical applications. $\mathrm{He}$ is currently finding a novel optical diagnosis tool for dentistry.

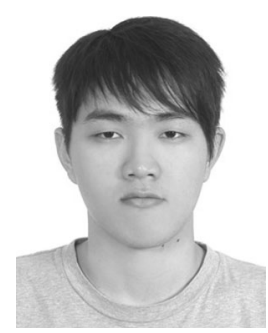

Chun-Yang Wang received the B.S. degree in physics from Fu Jen Catholic University, Xinzhuang, Taiwan, in July 2008. He is currently working toward the M.S. degree at Display Institute, National Chiao Tung University, Hsinchu, Taiwan.

His research interest includes biophotonics.

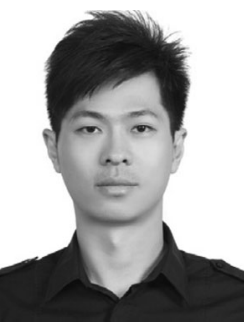

Chia-Wei Sun was born in Taiwan in 1975. He received the B.Sc. degree in electrical engineering from National Cheng Kung University, Tainan, Taiwan, in 1997, and the M.Sc. degree in biomedical engineering from National Yang-Ming University, Taipei, Taiwan, in 1999. He received the Ph.D. degree from the Institute of Photonics and Optoelectronics, National Taiwan University, Taipei, Taiwan, in 2003.

From 2003 to 2008, he was at the Medical Electronics and Device Technology Center, Industrial Technology Research Institute, where he was involved in clinical applications of biomedical optical imaging. In 2005 Summer, he joined the Computational Optics Group at University of Tsukuba, Ibaraki, Japan, as a Visiting Professor. He is currently an Assistant Research Fellow at the Biophotonics Interdisciplinary Research Center, National Yang-Ming University, and a concurrent Assistant Professor at the Department of Photonics, National Chiao Tung University, Hsingchu, Taiwan. He contributed to more than 40 peer-reviewed journal papers. His current research interests include diffuse optical tomography, near-infrared spectroscopy, optical coherence tomography, optics in brain science, and clinical applications based on biomedical optical imaging techniques. 